\title{
5G-Network-Based Connectivity and Data Platforms for Smart Cities- an Explorative Case Study of Drivers for Industry Transformation
}

\author{
Taina Tukiainen \\ School of Business, Aalto University \\ taina.tukiainen@aalto.fi
}

\author{
Lauri Paavola \\ School of Business, Aalto University
}

\begin{abstract}
Digital platforms, more detailed connectivity and increased availability of data are re-shaping markets. This is an opportunity for telecom companies to partner with other industries and offerings. We conduct an explorative case study to understand the connectivity and data platforms in smart cities. The contribution of our study is that it provides insights on the business models, platform control and competitive strategy in smart cities. Specifically, we find that the understanding of business models together with political, economic, social, environmental, technological and legal aspects are prerequisites to successful cases. Finally, we highlight the importance of co-creation and collaboration, as a means to tackle the challenges of platform localisations. Based on our findings we indicate promising aspects for future research in these fields.
\end{abstract}

\section{Introduction}

Our modern society and growing cities face great challenges nowadays, as an example to improve safety, energy efficiency, air quality, effectivity of transportation, and general quality of living. Cities have started to see the value of data when seeking to provide this. Data is needed for new services to enable sustainable economic growth and development in the city area and to meet the UN SDG's (Sustainable Development Goals). Current regulation of mobile networks and encrypted data traffic do not support properly the development of new data driven smart city services and business models.

Our study provides on the understanding of how fast 5G-network-based infrastructure can be utilized and data platforms optimized for the local needs of smart cities. 5G mobile networks are expected to change the current telecom operator business to become more local as the networks are based on small cells, i.e., high density of base stations.

The studied highly multi-disciplinary joint $5 \mathrm{G}$ $\mathrm{R} \& \mathrm{D}$ pilot project demonstrate developing new sharing business for digital smart city the data platform and operational model for the network, with the shared target to create an open ecosystem of citizens and companies to invent and create. This concept we will call 'neutral host'.

This neutral host concept is a relatively new phenomena in the area of data and telecommunications. The concept emerged in the mid 2000s from telecommunications infrastructure sharing operations [1]. Later, the concept diffused to the business literature [2] and furthermore to the science literature [3]. There have been several studies related to the concept recently, but it seems that the paradigm has not settled yet due to the fact that there are still several alternative definitions for the term neutral host [4]. Most of the recent scientific papers use the term neutral host when they refer to a network operator which maintains a small cell based mobile network for sharing purposes.

There is a need for the technology as the demand for connectivity capacity is expected to grow so high that dense network of $5 \mathrm{G} \mathrm{mmW}$ access points are a necessity. Also, the needed data collection, processing, storage, security and sharing and defined structures and processes are needed. To build one system enables regulations, GDPR and MyData rules to be agreed only once to the whole city area. To build a platform for active data-driven business and digital services meeting the needs in smart city and the technical elements but also best practices for safe and ethical use of data and sustainable business meeting the sustainable development goals (SDG) needs to take care.

Regarding data, Apple and Google are offering Global ecosystems which limit the data sharing. There are some operational models for local operators to offer, for example, wireless internet access for public. Typically, these are used inside large venues or other busy locations. They are usually deployed, maintained and operated by a third-party provider and they are designed to support the full range of MNO technologies. However, these solutions are providing only connectivity, not the platform and support for 
application developers and solution providers. For smart cities, bringing applications, new services, and broader and better connectivity is vital.

As it seems that the journey of neutral host technologies is just about to begin, we are seeing varying developments taking place around the world. Hence, in this study we ask the research question: What are the requirements for the successful technological development in smart cities?

\section{Theoretical background}

Several scholars have recently studied telecom or data business models and strategy related issues in smart cities [5]. Previous studies define the smart city from the perspective of technological development by describing that the smart city uses Internet of Things (IoT) devices to collect data and that ubiquitous connectivity services are available [6]. Furthermore, these studies envision that 5G-based small-cell networks will provide connectivity and platforms in cities via hosting companies operating the networks and these companies share the network infrastructure among all ecosystem stakeholders. The data and communications markets are significantly different and there are very few companies on the market that operates on both of these markets.

Since platforms play a central role in business ecosystems, platform management must be seen as an essential business ecosystem leadership capability. Internal platforms are within the boundaries of a single firm, while supply-chain platforms are among parties of supply chain, where firms in many cases are loosely coupled to industry platforms. This allows, that platform owner benefit the external innovation.

Platform management has turned out to be a crucial element in business development [8]. Efficient platform management allows rapid development of market share and revenue. Actually, platform leaders address the "needs of large, globally heterogeneous group of end users in a manner that would be prohibitively difficult for the platform core to do alone" [9]. Obviously, the connection between platform leaders and startups are vital, however, little is known about the strategic behavior of startups in relation to platform leaders.

A platform strategy should be understood as an emerging pattern where technology, applications, and organizations play complementary roles [10]. This means that the markets and competencies are essential to sustain growth, development and implementation of platform strategy. However, understanding and accepting the emerging character of platform strategy is not enough in order to achieve platform success. Researchers [11] [12] point out that a successful platform strategy for a firm in one part of the world, does not necessarily work in another part of the world. That is, a platform strategy is context dependent. As an example, a game developer would probably create its own internal platform that could work as a base for game creation. The games could then be distributed using global platforms like Apple iOS, Google Android or others. Such strategy may not be suitable for a startup in another line of business. Nevertheless, little is known about how startups manage such issues.

Scholars have studied how to gain and retain platform leadership. In the context of platforms, the business model comprises how a firm economically engages with external parties with the value proposition and its delivery.

As part of platform management, platform leaders also try to manage interdependencies by creating and applying rules of the game [13]. Such rules are for example implemented through the use of standardized interfaces like application domains with specific requirements or by offering products and complementary services to meet these specific requirements [14]. Rules of the game are necessity in many ecosystems [15]. However, there are also examples when software is used throughout and across various forms of industries, as in the case of data security, the software is boundary less in that context. The startups should therefore study the role of for example software in the current business, what role it should play, and how software collaboration could create a competitive advantage. The startups need to decide whether they should strictly follow the rules of the game, stretch the rules of the game, or create new rules [16].

The issue of Intellectual Property Rights (IPR) is also related to rules of the game. Researchers [17] stress that IPR play a governance role, they explain that independent software vendors need strong intellectual property rights and/or the possession of downstream complementary capabilities (brand image, downstream marketing, distribution, and service capability) in order to manage the relationship with platform leaders. The necessity of such measures can be questioned if the platform leader act with good ethics and focus on leveraging its partner's advantages. Simply put, some business ecosystem leaders can be trusted, others cannot. This line of argumentation indicates that managers in startups should therefore carefully study their business ecosystem from a "rules of the game" point of view. Based on the analysis they could develop dynamic capabilities and adapt to the level of trust inherent in the business ecosystem (-s). This should be considered to be a key issue since the development of IPR takes 
time and resources. However, startup behavior has not been studied from this point of view.

Moreover, nurturing of the business ecosystem is another dimension of creating rules of the game. Intel has nurtured their ecosystem by actively giving away IP and subsidizing competitive entry. Some researchers [18] propose that for Intel nurturing of complementary firms resulted in increased competition among complementary firms and a leadership advantage for Intel and describe nurturing of an ecosystem as a manipulation of the rules in the ecosystem - who can participate and in what way. The firms may create architectural advantage by stimulating competition in complementary products. Although the example of Intel only provides an example of nurturing through IPR, it must be stressed that nurturing also could take other forms. For example, startups may get access to knowledge resources, particular development resources or participate in partner programs. This line of argumentation indicates that, a startup who aspires to become platform leader, should early in its phase of business development map its potential complementors, and analyze, how these complementors may be nurtured. Because, as put by [19], "Within an ecosystem, firms must strive to take advantage of all available expertise and resources, which assumes they have access to them". However, little is known about such startup behavior.

In general, researchers [20] recommend managers to have strategic patience and build infrastructure to support innovation, and create an ecosystem that enables to develop creativity and cultivated taste for failure and chaos. As an example, Amazon [21] and Deutsche Telecom has demonstrated [22] this passion to their stakeholders. Deutsche telecom has invested in a T-lab, which is a research interface with universities and a partner development center. However, business ecosystem studies demonstrate no insight of how startups can draw such benefits of its related business ecosystem.

The relationship between business ecosystems and innovation is important since new business ecosystems are being formed around novel technologies [23]. Clearly innovations can be of various type of nature, anyway propose that organizations should adapt their innovation strategy to the ecosystem and form a good balance with various stakeholders.

\section{Methods}

This research is based on a four-year longitudinal study with 15 different telecommunications and data companies demonstrate aims to develop new sharing business for digital smart city and an open ecosystem of citizens and companies to invent and create in Europe.

For the purposes of answering the posed research question, we opt for a PESTEL analysis to understand the requirements behind the successful technological development. This PESTEL analysis (political, environmental, social, technological, economic, and legal) describes a framework of environmental factors used in the environmental scanning component of strategic management. It is part of an external analysis when conducting a strategic analysis or doing market research, and gives an overview of the different macro-environmental factors to be taken into consideration. It is a strategic tool for understanding market growth or decline, business position, potential and direction for operations.

While we acknowledge that the studied factors overlap in many aspects, the analysis offers us tools to capture important aspects behind the development. These factors represent by no means clear-cut entities that have created barriers for the studied cases; however, most of them overlap and create complex dynamics that steer the overall development. For example, political factors are likely to create tensions to all, technological choices, legal regulation as well as on society as a whole.

\section{Analysis}

\subsection{Within case analysis}

The European Commission has defined a smart city as "a place where traditional networks and services are made more efficient with the use of digital and telecommunication technologies for the benefit of its inhabitants and business". The key components of a smart city are inter-operability of systems, city-wide connectivity, and security. A 5G network featuring ultra-high speed, super low latency, and massive connectivity is crucial when building a smart city and operating its multiple services and products. The functioning of a smart city requires that a lot of data and also real time data - is collected from different networks of mobile devices, sensors, connected vehicles, and public places. This helps to better understand the behavioural patterns and demands of residents and respond with appropriate solutions. A smart city ecosystem can provide solutions or solve problems in the fields of traffic and parking, lightning, water consumption, recycling waste, crime prevention and detection, emergency responses, environmental monitoring relating for example to emissions, air pollution levels and providing digital city services. 
Building a smart city requires open data and other kinds of data sharing. In addition, citizen engagement and enhanced services for citizens are important [24]. However, practical and legal barriers stand in the road to the smart city. A massive amount of data is needed. Its usability increases if it is stored in one place instead of storing it in silos by many different service providers. Data usability increases when it is stored in a format usable in different systems. Data is usually more valuable if it can be traced to one particular person, because that way it is possible to learn behavioural patterns and adjust and offer services based on that information.

All this raises questions relating to data security and personal data protection. As $5 \mathrm{G}$ positioning is capable of measuring accurate locations, the use of smart and connected devices increases the risk to location data privacy. This risk is mitigated with the EU's General Data Protection Regulation and the ePrivacy Directive, which regulate the use of personal data. On the other hand, personal data regulation sets boundary conditions for legitimate use of personal data and can create some restrictions for use of data. Because personal data regulation is quite new, it seems unlikely that it will be changed in the relevant respects, at least in the near future. A flexible interpretation of legitimate interest and public interest as grounds for processing, added to taking data protection requirements into account even at the design state of services will enable development of the smart city and its services. An adequate level of personal data protection and citizens' trust in legal processing of data and data security raise willingness to share data with the Neutral Host (data market place) and take advantage of the many services that service providers in the smart city can make available. Informing data subjects about the purposes of data processing and how and why data is processed, as well as transparency of the functions of the controller, bear an important role in building data subjects' trust.

The requirement of transparency and responsibility also relates to provision of AI-driven products and services. The European regulatory approach to AI aims at balancing between sharing the risks of harm and damage caused by AI on the one hand, and promotion of innovation on the other hand. Operators in the Neutral Host ecosystem developing, deploying, or using AI-based solutions must closely follow the ongoing discussions and be prepared to comply with upcoming adjustments to existing legislation and any AI-specific legal instruments. As far as no special regulation exists, the basic rules and principles of tort law, contract law, and product liability law apply to damage caused by the use of AI.
Telecommunication regulation supports building and operating the $5 \mathrm{G}$ connectivity networks that smart city solutions require. Moreover, cybersecurity and OI regulation help to ensure secure and equal use of $5 \mathrm{G}$ networks for end-users. Even though the impact of EU regulation has increased in the telecommunication field, certain country-specific rules need to be paid particular attention when engaging in operative actions in the connectivity platform. For example, decisions on frequency allocation and spectrum licence conditions need to be considered separately for each smart city area. Moreover, the competent authorities for granting spectrum licence permission may differ between Member States. Rules of competition law are also of high importance as running 5G networks may entail more forms of cooperation among actors in the connectivity platform. For example, approval by competition authorities may also be needed for network sharing agreements and joint ventures between the parties involved.

One advantage of a smart city ecosystem is that it supports energy efficiency, the use of green energy and development of more environmentally friendly solutions in traffic. Kera area in the city of Espoo has been planned to become an international example area of the circular economy, with existing rail traffic and $5 \mathrm{G}$ technology enabling implementation of IoT and mobility solutions. We believe that in the future the requirements of the smart city for urban planning will be better taken into account, as this will also contribute to other goals considered important in society, such as sustainability and the circular economy.

While EU and national competition law do not pose any fundamental obstacle to the data platform or $5 \mathrm{G}$ connectivity aspects of smart cities, the various practices involved in establishing and operating them need to be evaluated carefully by the parties since they are capable of harming competition and can thus require justification. Moreover, some practices that might be involved, such as creation of joint ventures, must be notified to one competition law authority or several authorities for review before they can be implemented. At the moment, uncertainty prevails over how competition authorities and courts will treat some of these practices as no precedents exist in case law or guidance for the novel arrangements concerned (e.g., 5G connectivity offered as a Neutral Host). Moreover, the parties should monitor legislative work under way at EU and national level as that may in the future particularly affect how data platforms can be operated.

In conclusion, the current legislative framework provides a good basis for building a smart city and data marketplace. The most significant factor influencing development of the data marketplace is probably 
personal data protection regulation, which sets the boundary conditions for collection and use of data. To tackle this problem, it is important to be aware of regulation and recent interpretation in order to avoid over-cautious conclusions. The restrictions imposed by personal data protection regulation do not apply only to companies operating in Finland, but to all companies offering products and services to consumers in Europe. Similarity of the level of protection can be considered as an advantage, because it makes it easier for companies to offer their products and services for consumers in all EU countries. An adequate level of personal data protection can also become a competitive advantage.

\subsection{Cross-case analysis}

Our analysis focuses in the concept of the connectivity and sensor networks and a data platform, a data marketplace and a business model [24]. The connectivity and sensor network are based on a infrastructure that integrates connectivity and sensor technology in lighting poles. Poles represent a strategic infrastructure for smart city development, thus there is a global interest to develop smart light poles. The pole networks provide a platform for connectivity, computing, sensors and drones.

In order to develop and run global and local services and a data marketplace in a smart city platform, service providers need to collect and process personal data. The Neutral Host platform comprises several participating companies and organisations. We assume two different kinds of processing situations: one that relates to processing data for participating parties' own business purposes, the other relating to selling or sharing data in the Neutral Host data marketplace.

For the first possibility and considering the perspectives of different use-cases of the Neutral Host, the data controllers are business organisations and technology companies that take the initiative to implement smart city use-cases with the authority to determine the purposes and means of personal data processing. ${ }^{1}$

In the second scenario, the Neutral Host will become a controller because it is an individual body that determines the purposes and means of processing personal data. In this scenario the stakeholders deliver personal data for the NHP for its own purposes, namely to enrich, combine and sell data.

\footnotetext{
${ }^{1}$ According to Article 4(7) of the GDPR “"controller' means the natural or legal person, public authority, agency or other body which, alone or jointly with others, determines the purposes and means of the processing of personal data; where the purposes and
}

A successful future smart city produces and consumes data fluently. Currently, many data are isolated in silos that do not interact and opportunities for solving some of the pressing problems are lost. The success of future cities depends on their ability to share and use data that different activities and stakeholders, people and devices, infrastructures and authorities are producing. To make this happen, cities need improved connectivity and data transmission, intelligent data management and processing, the most efficient processing in edge and cloud, architectures for interoperability, data marketplaces and innovation of data-driven services.

The development will be iterative, but as with the development of any digital platforms, Neutral Host faces the chicken and egg strategy problem. It occurs when the value of the platform to data providers and data users is dependent on the number of participants on the other side. Data providers are not willing to put data on sales, if there are no data users, and data users are not using the platforms if there is no data available. Thus, the development needs investments to push either or both sides forward and initiate hopefully a positive feedback loop.

Neutral Host promotes creation of data markets and data business ecosystems where different stakeholders like citizens, enterprises, and authorities and data providers and service developers exchange data in different roles as sellers, buyers and facilitators. This requires implementation of data marketplaces that facilitate commercial transactions on the data products. The hypothesis is that by introducing data markets we motivate opening up existing data, productising of data, creating new data and taking data into use in data-driven services. This will pave the way for a society where the potential of existing data is used to benefit different actors in cities. The data marketplaces serve people e.g., citizens, service and business developers, data owners, and data users, but also machines via APIs in order to promote creation of automatic data economy where software components in various devices (mobile apps, vehicle software, building automation) search for, sell, buy and use relevant data without human intervention. This is especially relevant in $5 \mathrm{G}$ enabled real-time applications when there is no time to wait for human decisions, e.g., in autonomous traffic.

Neutrality means that Neutral Host serves all players equally aiming at synergetic implementations of the functions to save costs and to avoid multiple unnecessary implementations of the same function,

means of such processing are determined by Union or Member State law, the controller or the specific criteria for its nomination may be provided for by Union or Member State law". 
such as connectivity. Hosting means that it hosts all functions to provide the core of the data ecosystem functionality, however, enabling other surrounding implementations to flourish as the ecosystem grows, and other data platforms, data users, data providers and data marketplaces to integrate with the data ecosystem. Actually, integration is a crucial point, as Neutral Host is located in an environment where data are coming from local sources, but also from neighbouring and global sources. City contains local data, but also global data platforms and data marketplaces contain data about the city, such as weather or travelling services. Neighbouring cities may contain data about the city, especially in the areas between the cities, as well as the services used in the city might require data from neighbouring cities, e.g., environmental or infrastructure data related to navigation services. From a citizen viewpoint, a service used in one city should be available similarly when travelling to another city, without a need to reconfigure or install new software.

This presents the iterative development challenge and the dependability between different components of Neutral Host. In addition to data providers and data users in a two-sided platform, there are more stakeholders involved, and Neutral Host can be considered as a multi-sided platform including connectivity and sensor developers, data platforms and providers, data marketplaces, service developers and service end-users. One open question is how much subsidisation each side requires before the positive development cycle is created.

The iterative development of a Neutral Hostdriven data ecosystem requires that there is demand and users for sellable data. When connectivity is available sensors can be installed to produce sensor data of city. When data coming from sensors are available the value of data processing increases and introduces a motivation for developing data platforms and data markets that serve the data-driven services. When services create value there is extra motivation and demand to improve the connectivity network and install new sensors for new parameters or for new geographical areas, which again lead to new and improved data for new and improved services and additional value for end-users.

We introduce part of the motivation for this study. How political, economic, social, technological, environmental and legal factors affect the development within different timeframes? Do the factors have different effects on different components at different times? Can the analysis help in finding answers to the key question of the amount of subsidisation for each component?
Digitalisation means the process of transforming information processing into digital format produced and consumed by different organizations in the society and business. Digital data can more easily be shared, accessed and copied, and this is why it is a favoured way of preserving information for organizations around the world.

Digital platforms are businesses that enable and support interactions between distinct but interdependent groups of users (customers and suppliers). The platform acts as facilitator of exchanges of goods by using pricing strategies, and as participants co-create value between each other and create a "network effect" that means that a good or a service acquires more value to its user as more users adopt it. Network effects create self-reinforcing mechanisms that lead to market leadership, a large customer base economy of scale and the establishment of boundaries for other players.

Digitalisation creates new opportunities for $\mathrm{NH}$ data economy and for data business as larger part of smart city data is in digitized format ready to be published in data marketplaces. Many challenges remain to be solved though, e.g., how the data publishing is motivated and how the data quality, privacy, security and integrity are guaranteed.

Digital platforms and data marketplaces create more value from data in smart cities and will have a central role in smart city data economy and services.

Data business will provide more data available for actors in smart cities and allows creation of new kinds of applications and services that are based on commercial data products in smart cities. For example, applications such as digital twins, AR/VR applications, and applications that support decisionmaking can benefit from commercial data products that provide increasing volume of near real-time situational data of city environments.

Some researchers discuss the key aspects that are related to a smart city: Smart Economy (Competitiveness), Smart Governance (Citizen Participation), Smart People (Social and Human Capital), Smart Mobility (Transport and ICT), Smart Environment (Natural Resources), and Smart Living (Quality of life). The data business can relate to all these aspects. For example, implementation of these aspects in smart cities will produce demand, suppliers, and users for commercial data products.

\section{Interpretation and discussion}

According to UN reports, $56 \%$, some argue over $70 \%$ of the global population resides in urban areas of the world. The only way for cities to improve the quality of life as the world's population grows at a 
tremendous rate is to discover and implement new technologies in different sectors. One such type of technologies are the innovations such as Smart cities and Neutral Host networks (NHNs).

In a nutshell, an NHN is a third-party cellular network in charge of providing localised mobile coverage solutions to a wide range of national mobile network operators (MNOs). The NHN itself determines whether their commercial mobile network solutions are paid or free of charge or whether they belong to the MNO's bands or a dedicated NHNowned spectrum. In European regions, these innovations are expected to be implemented mainly with-in transportation and mobility sectors to increase functionality and citizen engagement.

Based on our research, one outcome of integrating these technologies would be the reduction of the use of personal cars, as the cities are going to provide more reliable and pleasant public transportation. With improvements to shared mobility, cities are going to benefit in terms of environmental changes and higher satisfaction among the citizens. However, it is important to address that neutral host technologies also play a significant role in various other industries, including media and entertaining, the automotive industry, logistics, safety, drone technology, healthcare industries, and energy, among others.

Neutral host technologies are bound to increase network capacities and processing capabilities, which is going to lead to more advanced data processing systems and thus a higher degree of data collection. As big data continues to develop on a larger scale, technologies such as AI and machine learning will benefit from the increased capacity and data variety. The increase in data processing volume is one of the bases for EU's key strategies including the European Data Economy and the Digital Single Market. As a result, API ecosystems will support data economy development and provide additional data for this cause.

There are many data-driven value propositions worth mentioning, including information and knowledge or a non-data product or service. Data business models expand through various uses, from models for data suppliers and data users to data facilitators and data sharing technologies. Potential value of data will remain untapped as long as data remains fragmented in isolated systems. Opening up the door to this new world of data could solve a variety of smart city challenges the world is currently dealing with. In order to make smart cities a reality, business models must put together a smart city data economy.

The data-driven value proposition can be data, information/knowledge, or a non-data product or service [39]. There exist data business models for data users, data suppliers, and data facilitators and emerging data sharing technologies such as data marketplaces and payment solutions for data-driven business. However, data is still fragmented in isolated systems and lot of potential value remains unrealized as all the data cannot be utilized to tackle various challenges of smart cities. Going beyond state-of-theart requires new business models to develop smart city data economy that creates new value from data, boosts the use of private, public, open and commercial data, and opens up currently hidden data storages for new applications. Solutions and technologies for selling and buying of data products are needed as well as business expertise and funding to enable creation of new business models and new value from data in various business cases.

Our research emphasizes that data economy requires data networks that are fair, balanced and lawful in their processing of data [27]. These data networks must be just and impartial toward their members, ensure that the right of third parties are not infringed, and make sure that EU level and applicable national data protection regulations are followed in processing of personal data. The data network must identify and manage risks associated with the sharing and processing of data and ensure compliance with relevant competition legislation and that the data network will not have a negative impact on market competition and consumers [24]. As a result, it is easier for companies to define rulebooks for data networks, to assess requirements imposed by applicable legislation and contracts appropriately, and to adopt practices promoting the use of data and management of risks [24].

For example, the provided templates assist companies to establish contractual frameworks for their data networks to define the legal relations within their data networks and to set out general terms and conditions for data sharing agreements.

This will create a new value from data which will enable new technologies and applications. The currently un-used data storages can significantly boost the speed of smart city development. Other solutions needed for this cause are technologies for exchanging, that is, selling and buying data products. Of course, the implementation of these technologies will require business expertise and funding in order to form the new business models. With da-ta-business and communications- businesses being completely different, a neutral-host business model, if established, would be operating in two separate markets. This could be a potential block on the road, although there are ways to work around it. After all, data and communications businesses, even though fundamentally different, are both platform-based. 
Each platform would need a certain volume of customers to cover the costs of the new operations. With that in mind, the geographical location where the natural host would be operating would have to be vast enough to provide the required customer volume.

Moreover, it is important to address the categorization of the ownership structures. There are two extreme ends - one; which is a neutral host or a public stock-listed company, and the other; a nonprofit organization that takes care of non-monetary objectives. There are, however, several minor categories between these two. With that in mind, we must take into consideration that the ecosystem will vary if operated by different owner-base-neutral-hosts. The concept of ownership can have a significant impact on the implementation of these categories.

The second categorization implies that certain companies choose similar strategies based on their historical background. This piece of information helps neutral hosts determine how to run a business and which growth path to choose. We have also covered the traits and strategies of neutral host companies in regards to their use of data. The goal behind these reports is to understand how neutral host companies act in their ecosystems. In our re-search of the given matter, we have discovered that neutral host companies have numerous businesses possibilities that could be beneficial to both citizens and the government in a smart city ecosystem.

As we have discussed in this study, it is important to take into consideration all the legal aspects that go into developing a neutral host business. Collecting and processing personal data of any kind requires compliance with certain privacy laws and regulations. The neutral host company also has to comply with the requirements of competition law, which usually requires each planned joint venture to be announced to the competition authorities. Throughout the entire process of ensuring that the smart city ecosystem is in full compliance with the law, it is important to keep in mind that legal matters are constantly changing and developing. This makes pinpointing specific questions and answers difficult, as we are dealing with a new legal environment that is constantly evolving.

The purpose behind regulating these activities is to enable implementation of smart cities in the future. To do so, certain regulatory procedures will have to be lightened, as they will no longer be applicable to $5 \mathrm{G}$ implementation. This need has already been acknowledged in Finland and at the EU level. There is no doubt that there will be countless new concerns regarding the legal side of enabling smart cities. However, legislators and regulators will have to work together to find a balance between restrictions and potential benefits. As we have for example seen in this report, with regard to relevant frequencies that remain unallocated $(26 \mathrm{GHz})$, the means of allocating them will be crucial. It remains to be seen how they are allocated.

Despite all potential concerns, there is a way for the concept of a Neutral Host to be implemented under the current regulatory framework. According to the current laws, a $5 \mathrm{G}$ system can legally operate as long as citizen's data and privacy are fully secured. Another requirement is that all elements needed for bringing a smart city to life are shared and used in the most effective way possible. While we do expect the development of new and lighter reforms that will allow smart cities to function on a larger scale, the influence of the Neutral Host won't be estimated until later on in the future. Until then, Neutral Host can be developed through existing legal framework, as long as all privacy requirements are taken into consideration.

\section{Conclusions}

In this study, we have analysed macroenvironmental factors of the Neutral Host concept in the domain of a smart city where data are produced and consumed fluently for the benefit of city functions and citizens' wellbeing. A Neutral Host is a new business and the analysis includes factors that may have impact on its performance including Political, Economic, Social, Technological, Environmental and Legal factors according to the PESTEL framework. Neutral Host is an organization that operates various functions of a smart city data ecosystem, such as connectivity and sensor networks, computing resources and data processing, and data markets and innovation of data-driven services.

Neutral Host promotes creation of data markets and data business ecosystems where different stakeholders like citizens, enterprises, and authorities and data providers and service developers exchange data in different roles as sellers, buyers and facilitators. This requires implementation of data marketplaces that facilitate commercial transactions on the data products. The hypothesis is that by introducing data markets we motivate opening up existing data, productising of data, creating new data and taking data into use in data-driven services. This will pave the way for a society where the potential of existing data is used to benefit different actors in cities. The data marketplaces serve people e.g., citizens, service and business developers, data owners, and data users, but also machines via APIs in order to promote creation of automatic data economy where software components in various devices like mobile apps, vehicle software, building automation search for, sell, 
buy and use relevant data without human intervention. This is especially relevant in 5G enabled real-time applications when there is no time to wait for human decisions, e.g., in autonomous traffic.

Building such environment requires open data and other kinds of data sharing. In addition, citizen engagement and enhanced services for citizens are important. However, political, economic, environmental and legal drivers steer the development of a smart city. A massive amount of data is needed. Its usability increases if it is stored in one place instead of storing it in silos by many different service providers. Data usability increases when it is stored in a format usable in different systems. Data is usually more valuable if it can be traced to one particular person, because that way it is possible to learn behavioural patterns and adjust and offer services based on that information.

\section{References}

[1] Muir, S., Chapin, J., Bose, V., \& Steinheider, J., 2006. Distributed antenna systems plus software radio: Range extension and other benefits, In Vanu, Inc., Software Defined Radio Technical Conference, Orlando, FL.

[2] Rayal, F. 2013, LTE-Advanced, 3.5 GHz, Small Cells and Neutral Host Services: A Powerful Mix to Abundantly Increase Network Capacity, Frank Rayal Blog, viewed 4 February 2020.

[3] Hadi, M. U. 2015, Extending the benefits of LTE to unlicensed spectrum, 2015 International Conference on Information and Communication Technologies (ICICT) (pp. 1-3). IEEE.

[4] Paglierani, P., et al., 2019. Techno-economic analysis of $5 \mathrm{G}$ immersive media services in cloud-enabled small cell networks: The neutral host business model: Providing techno-economic guidelines for the successful provision of $5 \mathrm{G}$ innovative services in small cell networks. Transactions on Emerging Telecommunications Technologies, e3746.

[5] Perätalo, S., \& Ahokangas, P. 2018. Toward Smart City Business Models. Journal of Business Models, 6(2), 65-70.

Walravens, N., \& Ballon, P. (2013). Platform business models for smart cities: from control and value to governance and public value. IEEE Communications Magazine, 51(6), 72-79.

[6] Strohbach, M., Ziekow, H., Gazis, V., \& Akiva, N. (2015). Towards a big data analytics framework for IoT and smart city applications. In Modeling and processing for next-generation big-data technologies (pp. 257-282). Springer, Cham.

Kibria, M. G., Villardi, G. P., Nguyen, K., Liao, W. S., Ishizu, K., \& Kojima, F. (2017). Shared spectrum access communications: A neutral host micro-operator approach. IEEE Journal on Selected Areas in Communications, 35(8), 1741-1753.

[8] Gawer, A. 2009. Platform Dynamics and Strategies: From Products to Services. In A. Gawer (Ed.),
Platforms, Market and Innovation:45-76. Cheltenham, UK: Edward Edgar Publishing Limited.

[9] Wareham, J., Fox, P., Cano, G., \& Li, J. 2014. Technology Ecosystem Governance, Organization Science, 25(4): 1195-2015.

[10] Rong, K., Hu, G., Lin, Y., Shi, Y., \& Guo, L. 2015. Understanding Business Ecosystem Using a 6C Framework in Internet-of-Things-Based Sectors. International Journal of Production Economics, 159, 41-55. https://doi.org/10.1016/j.ijpe.2014.09.003

[11] Gawer, A. 2009. Platform Dynamics and Strategies: From Products to Services. In A. Gawer (Ed.), Platforms, Market and Innovation: 45-76. Cheltenham, UK: Edward Edgar Publishing Limited; Kenney, M., \& Pon, B. 2011. Structuring the Smartphone Industry: Is the Mobile Internet OS Platform the Key? Journal of Industry, Competition and Trade, 11(3): 239-261. https://doi.org/10.1007/s10842-011-0105-6)

[12] Hänninen, M., \& Paavola, L. 2020. Digital Platforms and Industry Change. In H. Lehtimäki et al. (eds.), Society as an Interaction Space, pp. 213-226. Springer, Singapore.

[13] Jansen, S., \& Cusumano, M. A. 2013. Defining Software Ecosystem: A Survey of Software Platforms and Business Network Governance. In S. Jansen, M. A. Cusumano, \& S. Brinkkemper (Eds.), Software Ecosystems: Analyzing and Managing Business Networks in the Software Industry: 13-28. Cheltenham, UK: Edward Edgar Publishing Limited.

[14] Mazhelis, O., Luoma, E., \& Warma, H. 2012. Defining an Internet-of-Things Ecosystem. In S. Andreev, B. Balandin, \& Y. Koucheryavy (Eds.), Internet of Things, Smart Spaces and Next Generation Networking: 1-14. Berlin: Springer Berlin Heidelberg.

[15] Schumpeter, J. A. 1942. Capitalism, Socialism and Democracy. New York: Harper \& Brothers.

[16] Ceccagnoli, M., Forman, C., Huang, P., \& Wu, D. J. 2012. Co-Creation of Value in a Platform Ecosystem: The Case of Enterprise Software. MIS Quarterly, 36(1): 263-290.)

[17] Jacobides, M. G., Knudsen, T., \& Augier, M. 2006. Benefiting from Innovation: Value Creation, Value Appropriation and the Role of Industry Architectures. Research Policy, 35(8): 1200-1221.

[18] Isckia, T. 2009. Amazon's Evolving Ecosystem: A Cyber-Bookstore and Application Service Provider. Canadian Journal of Administrative Science, 26(4): https://doi.org/10.1002/cjas.119p. 335),

[19] Iyer, B., \& Davenport, T. H. 2008. Reverse Engineering Google's Innovation Machine. Harvard Business Review, 86(4): 58-69.

[20] Isckia, T. 2009. Amazon's Evolving Ecosystem: A Cyber-Bookstore and Application Service Provider. Canadian Journal of Administrative Science, 26(4): 332-342. https://doi.org/10.1002/cjas.119

[21] Rohrbeck, R., H lzle, K., \& Gemünden, H. G. 2009. Opening Up for Competitive Advantage: How Deutsche Telekom Creates an Open Innovation Ecosystem. R\&D Management, 39(4): 420-430 
[22] Kim, H., Lee, J.-N., \& Han, J. 2010. The Role of IT in Business Ecosystems. Communication of the ACM, 53(5): 151-156.

[23] Adner, R., \& Kapoor, R. 2010. Value Creation in Innovative Ecosystems: How the Structure of Technological Interdependence Affects Firm Performance in New Technology Generation. Strategic Management Journal, 31(3): 306-333.

[24] Pitkänen et al., 2019.Rulebook for Fair Data Economy Rulebook Template for Data Networks. Sitra, 2019. 